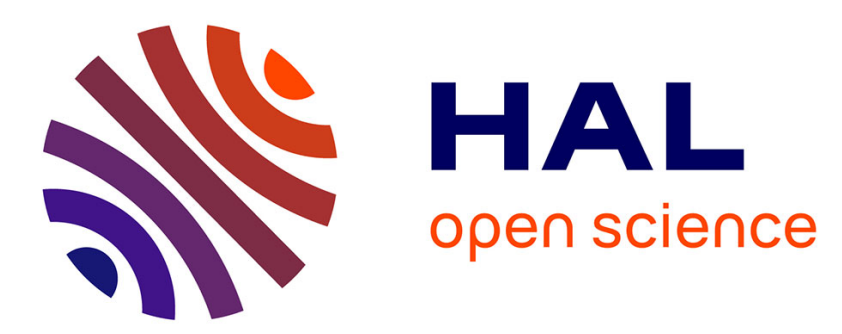

\title{
Energy Exchange between Femtosecond Laser Filaments in Air
}

Yi Liu, Magali Durand, Shihua Chen, Aurélien Houard, Bernard Prade, Benjamin Forestier, André Mysyrowicz

\section{> To cite this version:}

Yi Liu, Magali Durand, Shihua Chen, Aurélien Houard, Bernard Prade, et al.. Energy Exchange between Femtosecond Laser Filaments in Air. Physical Review Letters, 2010, 105 (5), pp.055003. 10.1103/PhysRevLett.105.055003 . hal-00510593

\section{HAL Id: hal-00510593 \\ https://hal-polytechnique.archives-ouvertes.fr/hal-00510593}

Submitted on 19 Aug 2010

HAL is a multi-disciplinary open access archive for the deposit and dissemination of scientific research documents, whether they are published or not. The documents may come from teaching and research institutions in France or abroad, or from public or private research centers.
L'archive ouverte pluridisciplinaire HAL, est destinée au dépôt et à la diffusion de documents scientifiques de niveau recherche, publiés ou non, émanant des établissements d'enseignement et de recherche français ou étrangers, des laboratoires publics ou privés. 


\title{
Energy Exchange between Femtosecond Laser Filaments in Air
}

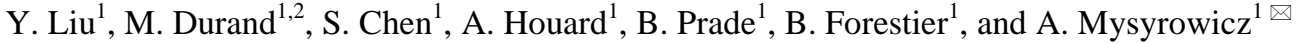 \\ 1. Laboratoire d'Optique Appliquée, ENSTA ParisTech, Ecole Polytechnique, CNRS UMR 7639, Palaiseau, 91761, France \\ 2. Département d'Optique Théorique et Appliquée, ONERA, 91761 Palaiseau, France \\ $\triangle$ E-mail address: andre.mysyrowicz@ensta.fr.
}

\begin{abstract}
We report on the energy exchange between femtosecond laser filaments in air. A traveling plasma grating formed at the intersection of the filaments is proposed to explain the energy transfer. In this moving plasma grating mediated mechanism the laser energy transfers from the lower frequency pulse to its higher frequency partner, while in a traditional Kerr nonlinearity mediated grating, energy transfer occurs in the opposite manner. Based on this mechanism, we demonstrate an energy transfer ratio as high as 50 percent for pulses with energy of several millijoules.
\end{abstract}

PACS numbers: 52.38.Hb, 42.65.Jx. 42.65.Sf

Propagation of powerful femtosecond $\left(10^{-15} \mathrm{~s}\right)$ laser pulses in air gives rise to a series of spectacular effects. As it propagates, the light pulse self focuses due to the change of refractive index it induces in the medium. For a pulse power exceeding a critical value $P_{\text {cr }}(\sim 5 \mathrm{GW}$ in air for $\lambda=800 \mathrm{~nm})$, diffraction is unable to arrest the beam self-focusing. The intensity in the beam center increases progressively with distance until it starts ionizing air molecules. Once created, the plasma induces a negative change of refractive index, an effect leading to beam defocusing. The ensuing dynamic competition between self-focusing and plasma defocusing is responsible for filamentation, a process where part of the beam maintains a very high intensity over long distances leaving in its trail a column of weakly ionized plasma [1,2].

A large part of the fascination with the process of filamentation is due to its potential for applications. The use of filaments has been proposed and/or demonstrated for various applications ranging from remote sensing of atmospheric pollution [3], recognition of distant targets by Laser Induced Breakdown Spectroscopy [4, 5], stand off THz illumination [6, 7], to triggering and guiding of electric discharges and even lightning [8]. For all these applications, control and optimization of the filament characteristics are desired. This can be done to a certain extent by modifying the laser beam characteristics before propagation $[9,10]$. In the last two years, more subtle ways to control filaments have been discovered. One concept relies on molecular lensing: the spontaneous revivals of the refractive index in air induced by a precedent femtosecond pulse can deviate or destroy subsequent filaments $[11,12]$. In another approach, the use of an intense Airy beam leads to a curved trajectory of the filament [13].

Here, we report on another approach to control filament properties, namely the possibility of exchanging energy between two femtosecond laser filaments in air. This effect should allow their regeneration and increase their length. Energy exchange between powerful femtosecond laser beams in air has been pioneered by Bernstein et al. who showed that two beams crossing at grazing angle could exchange up to $7 \%$ of their energy [14]. The exchange reported here, which has a different origin, has an efficiency up to $50 \%$ and is also observed at large crossing angle.

The physics of the process is captured in figure 1. Two laser pulses with power $P>P_{\text {cr }}$ of slightly different frequencies, form two intersecting plasma filaments in air (see Fig. $1 \mathrm{a}$ ). Fig. $1 \mathrm{~b}$ is actually a photo of the characteristic luminescence emanating from two such crossing plasma channels [15]. The fields of the optical pulses with wave numbers $\vec{K}_{1}$ and $\vec{K}_{2}$ interfere and give rise to a spatially periodic pattern $d=\lambda /(2 \sin (\varphi / 2))$, see Fig. 1 c. With a frequency difference of $\Delta \omega=\omega_{1}-\omega_{2}$, this interference pattern moves in the direction of $\vec{K}_{g}=\vec{K}_{1}-\vec{K}_{2}$ with phase velocity $v_{p}=\Delta \omega /\left|\vec{K}_{g}\right|$. The interference field leads in turn to the formation of traveling gratings via the nonlinear response of the medium.

In general, the nonlinear response of a transparent medium to an intense laser field consists of an instantaneous and/or a retarded change of the refractive index. It is only the retarded response that can result in energy exchange because it induces a spatial phase shift between the laser intensity interference and the refractive index modulation [16]. In the case where the nonlinear part of the refractive index follows the Debye relaxation, the nonlinear contribution of the refractive index reads $\Delta n(\vec{r}, t)=\frac{n_{2}}{\tau} \int_{-\infty}^{t} \exp \left(\frac{t^{\prime}-t}{\tau}\right) I\left(\vec{r}, t^{\prime}\right) d t^{\prime}$. From the wave propagation equation, the intensity variation of beam 1 (or 2) can be expressed as: $\frac{d I_{i}}{d z}=\mp \frac{2 \omega n_{2} I_{i} I_{j}}{c} \frac{\Delta \omega \tau}{1+(\Delta \omega \tau)^{2}}(i, j=1,2$ and $i \neq j)$, where $\tau$ and $n_{2}$ are the nonlinear response time and the nonlinear refractive index respectively [16]. In this case, two features can be found from above: (a) a positive refractive index response $\left(n_{2}\right)$ will induce laser energy flow from higher frequency to lower frequency pulses, 
while a negative $n_{2}$ will induce energy transfer in the opposite manner. (b) To achieve energy exchange, the interacting pulses must have different frequencies. The frequency difference dictates the direction of energy flow.

Now, we consider more specifically two retarded nonlinear responses in air, the change of refractive index due to the rotational Raman effect, and that induced by the formation of a plasma. For the Raman response of the molecule, the nonlinearity is related to the laser intensity as $\Delta n_{\text {Raman }}(\vec{r}, t)=n_{2} \int_{-\infty}^{t} R\left(t-t^{\prime}\right) I\left(\vec{r}, t^{\prime}\right) d t^{\prime}$. The function $R(\mathrm{t})$ mimics the molecule response with a characteristic time $\Gamma$ and frequency $\omega_{\mathrm{R}}$ : $R(t)=R_{0} \exp (-\Gamma t) \sin \left(\omega_{R} t\right)$, where $R_{0}(t)=\left(\Gamma^{2}+\omega_{R}^{2}\right) / \omega_{R}$. In air for a pulse at $\lambda=800 \mathrm{~nm}, \Gamma^{-1}=70 \mathrm{fs}$ and $\omega_{\mathrm{R}}=1.6 \times 10^{13} \mathrm{~Hz}[1,11]$. In Fig. $2 \mathrm{a}$ and $\mathrm{b}$, we plot the calculated temporal evolution of the laser intensity and the nonlinear index modulation as a function of position $x$ at propagation distance $z=0$ (see Fig. $1 \mathrm{c}$ ). In the simulation, the following realistic parameters were chosen: laser intensity in the filament $5 \times 10^{13} \mathrm{~W} / \mathrm{cm}^{2}$, frequency difference $\Delta \omega=10 \mathrm{THz}$ (see below) and crossing angle $2^{\circ}$. The interference pattern and index grating both move in the direction of $\vec{K}_{g}$. In Fig. $2 \mathrm{~d}$, the intensity (blue line) and the refractive index modulations (black line) at time $=-20 \mathrm{fs}$ are plotted. We note a spatial phase shift between the two modulations (of about 1.2 $\mu \mathrm{m})$, which is essential for possible energy exchange.

For the plasma generation process, the retarded refractive index change is related to the plasma density by $\Delta n_{\text {plasma }} \approx-\rho(\vec{r}, t) / 2 \rho_{c r}$, where $\rho_{c r}=1.7 \times 10^{21} \mathrm{~cm}^{-3}$ is the critical plasma density for $800 \mathrm{~nm}$ pulse and $\rho(\vec{r}, t)=\int_{-\infty}^{t} \sigma_{8} \rho_{m o l} I^{8}\left(\vec{r}, t^{\prime}\right) d t^{\prime}$ is the plasma density [1, 2]. The terms $\sigma_{8}$ and $\rho_{\text {mol }}$ are the multiple photon ionization cross section and the density of the neutral oxygen molecules. The calculated evolution of the moving refractive index grating at $z=0$ is presented in Fig. 2 c. The grating corresponds here to a free electron density modulation $[17,18]$. At the end of the pulse overlap, the plasma grating stays at rest and remains unchanged because its decay time is much larger than the time scale considered here. Fig. $2 \mathrm{~d}$ shows the spatial distribution of the plasma grating at time $=-20 \mathrm{fs}$ (red line). A spatial phase shift of nearly $180^{\circ}$ with respect to the driving interference pattern is apparent, due to the negative refractive index change. As a result, the energy is expected to flow now from the low frequency pulse to its high frequency partner.

In our experiment, we use a laser delivering pulses of $\tau_{0}=45 \mathrm{fs}$ duration centered at $800 \mathrm{~nm}$ with energy of up to $15 \mathrm{~mJ}$. To obtain two laser pulses with controllable difference in their instantaneous frequency, a linear chirp is impressed to the laser pulse before splitting it into two replicas. If pulse 1 is delayed with respect to pulse 2 by $\tau_{\mathrm{d}}$, the difference of the instantaneous frequencies is $\Delta \omega=-C \tau_{d} / 2 \tau_{p}^{2}$ [19]. Here, $\tau_{\mathrm{p}}$ is the chirped pulse duration with the chirp parameter $C= \pm \sqrt{\left(\tau_{p} / \tau_{0}\right)^{2}-1}$. At a given time delay $\tau_{\mathrm{d}}$ the two pulses possess a constant frequency difference during their time overlap.

Figure 3 shows results obtained in air and in Argon at 1 bar pressure, using a crossing angle of $\varphi=0.9^{\circ}$. A linear positive chirp (i.e. an increase of instantaneous frequency from the leading to trailing edge of the pulse) $C$ $=+5$ was impressed to the initial pulse yielding a pulse duration of $\tau_{p}=220 \mathrm{fs}$. To reduce the distance required for beam collapse, the two chirped pulse replica, both vertically polarized, were focused by two lens of $f=2000$ $\mathrm{mm}$. Above $P_{\mathrm{cr}}$, filaments were formed with a length of the plasma column up to $150 \mathrm{~mm}$ at $P=15 \mathrm{GW}$. Turning the attention to air first, we observe two different regimes. At lower power $P=0.39 P_{\text {cr }}$ (Fig. 3 a), transfer of energy is always directed from the high frequency beam to the low frequency beam. This is similar to the observation by Bernstein et al. and can be assigned to a grating induced by the rotational molecular response of the optical Kerr effect [14]. It would be interesting to explore this regime of energy exchange with very short pulses. One then expects an additional contribution from the vibrational Raman response of the molecules [20]. At higher incident laser power $P=3.5 P_{\text {cr }}$ (Fig. $3 \mathrm{~b}$ ), the energy transfer changes direction. This new regime is a genuine energy exchange between filaments in the sense that it occurs once plasma channels are formed along the paths of beams 1 and 2. In Fig. 3 c, the maximum energy exchange ratio defined as $S=\left(E_{1}-E_{2}\right) /\left(E_{1}+E_{2}\right)$ at positive $\tau_{\mathrm{d}}$ is plotted as a function of incident power, where $E_{1}$ and $E_{2}$ are the energy of the first and second pulses after exchange. The turning point between the two regimes occurs at a power close to $P_{\mathrm{cr}}$. Also, we point out that with negatively chirped pulses in both regimes the energy transfer is reversed between pulse 1 and 2 (for example, in fig. 3(a) for $\tau_{\mathrm{d}}<0$ the laser energy now transfers from pulse 2 to pulse 1 ).

With argon (see Fig. 3 d, e, and f), we notice the absence of energy exchange until the laser power reaches $P_{\mathrm{cr}}$. This is easily understood since there is no rotational Raman effect in an atomic gas. As expected, we find that energy exchange always occurs with a negative ratio $S$ (energy transfer from the lower frequency to the higher frequency pulse). With the increase of the laser power, $S$ increases almost linearly up to $12 \%$. 
Because the plasma grating is composed of a weakly ionized gas, a higher refractive index contrast can be expected with a higher intensity in the filament, allowing for more efficient energy exchange. It is possible to achieve this in short filaments by using more converging initial beams [21]. In Fig. 4 a, the exchange ratio $S$ is presented for focal lens of $f=125 \mathrm{~mm}$. It is seen that the ratio $S$ can reach $50 \%$ for pulses with initial power $P \sim$ $3 P_{\mathrm{cr}}$. In that case, the plasma column length is about $5 \mathrm{~mm}$. As an example, the variation of beam energy is shown in Fig. $4 \mathrm{~b}$ as a function of pulse delay (frequency difference $\Delta \omega$ ) for $P=1.5 P_{\mathrm{cr}}$.

Finally, the exchange ratio was also studied as a function of the effective interaction length, which was varied by changing the crossing angle while keeping the same focal length $f=300 \mathrm{~mm}$. The results are presented in Fig. $4 \mathrm{c}$, with the numbers on the top of each point indicating the corresponding crossing angle. As expected, the efficiency increases for longer interaction lengths.

In conclusion, we have shown that it is possible to exchange efficiently laser energy between filaments in air. The source of this novel energy transfer is a traveling plasma grating, which is applicable not only to Raman active molecular gases but also to monatomic noble gases. The magnitude and direction of energy flow from one beam to the other can be controlled by detuning the frequency of the two filamentation pulses. We believe this mechanism can be useful to remotely replenish filaments.

We acknowledge fruitful discussions with Professor V. Tikhonchuk and Dr C. Arnold and B. Zhou. This work has been partially supported by DGA. S. C. appreciates support from the National Science Foundation of China through Grant 10874024.

\section{REFERENCES:}

[1] A. Couairon and A. Mysyrowicz, Phys. Rep. 441, 47 (2007).

[2] S. L. Chin et al., Can. J. Phys. 83, 863 (2005).

[3] J. Kasparian et al., Science 301, 61 (2003).

[4] K. Stelmaszczyk et al., Appl. Phys. Lett. 85, 3977 (2004).

[5] H. L. Xu et al., Appl. Phys. B 87, 151 (2007).

[6] C. D’Amico et al., Phys. Rev. Lett. 98, 235002 (2007).

[7] Y. Liu et al., Phys. Rev. Lett. 99, 135002 (2007).

[8] M. Rodriguez et al., Opt. Lett. 27, 772 (2002).

[9] G. Fibich et al., Opt. Express 14, 4946 (2006).

[10] P. Sprangle, J. R. Penano, and B. Hafizi, Phys. Rev. E 66, 046418 (2002).

[11] J. F. Ripoche et al., Opt. Commun. 135, 310 (1997).

[12] S. Varma, Y. -H. Chen, and H. M. Milchberg, Phys. Rev. Lett. 101, 205001 (2008).

[13] P. Polynkin et al., Science 324, 229 (2009).

[14] A. C. Bernstein et al., Phys. Rev. Lett. 102, 123902 (2009).

[15] H. L. Xu et al., Chem. Phys. 360, 171 (2009).

[16] R. W. Boyd, NONLINEAR OPTICS, third edition, Academic Press.

[17] Z. -M. Sheng, J. Zhang, and D. Umstadter, Appl. Phys. B 77, 673 (2003).

[18] S. Suntsov et al., Appl. Phys. Lett. 94, 251104 (2009).

[19] A. Dogariu et al., J. Opt. Soc. Am. B 14, 796 (1997).

[20] E. Nibbering et al., Phys. Rev. Lett. 68, 514 (1992).

[21] F. Théberge et al., Phys. Rev. E 74, 036406 (2006). 


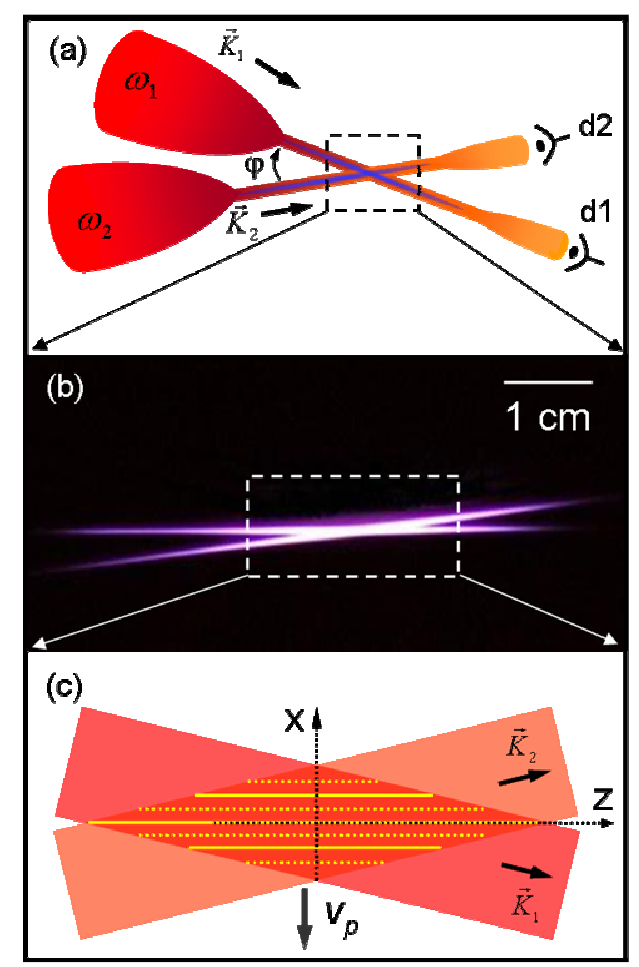

Fig. 1. Schematic experimental configuration with two interacting filaments. (a) Two focused pulse replica propagate from left to right and form two interacting filaments crossing under angle $\varphi$. The laser polarizations are orthogonal to the plane formed by the two beams. The pulse energies after interaction are measured by detectors $\mathrm{d} 1$ and $\mathrm{d} 2$. (b) Photo of two interacting filaments, displaying the characteristic near UV luminescence of $\mathrm{N}_{2}$ in air following ionization. (c) Schematic representation of the magnified area where the two plasma columns overlap, showing the interference pattern of the two laser fields traveling with a velocity $v_{p}$. The center of the overlapping area is chosen as the origin of the $x$ and $z$ axis.
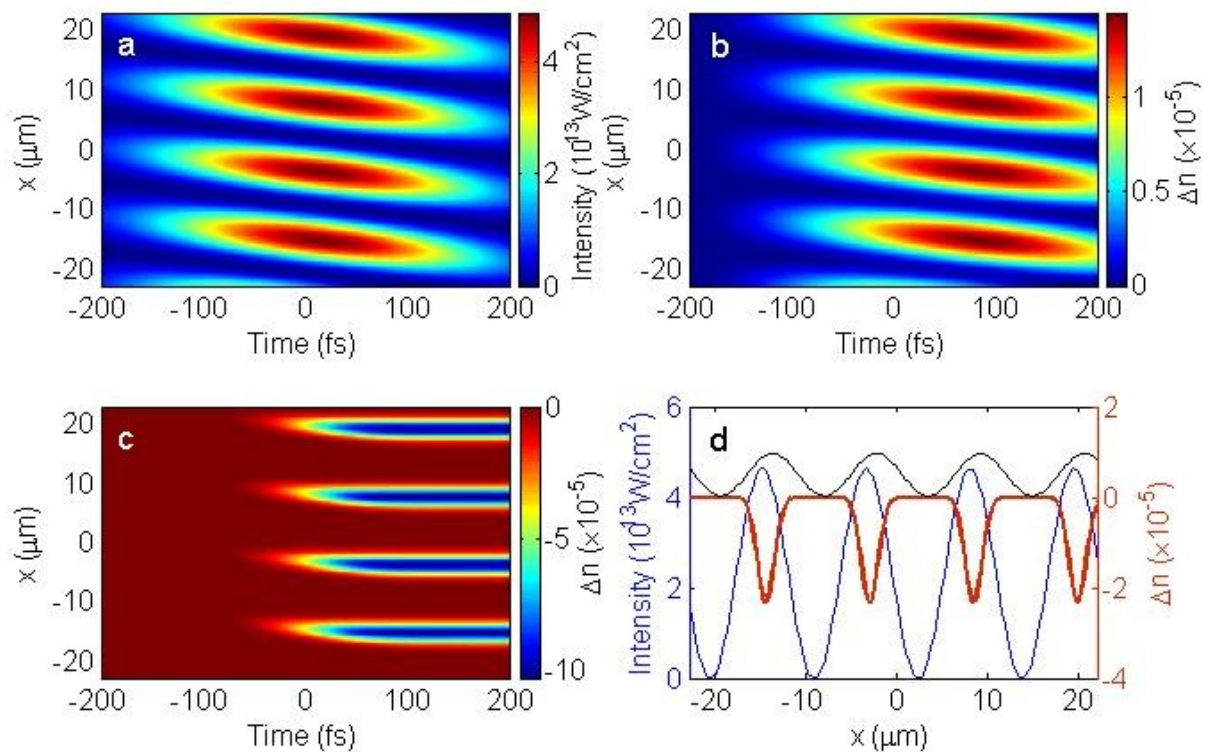

Fig. 2. Temporal and spatial distribution of laser intensity and refractive index modulation in the interaction region. (a), Temporal evolution of the laser interference intensity at $z=0$. Chirped pulse 2 of duration $200 \mathrm{fs}$ is delayed by $100 \mathrm{fs}$ with respect to chirped pulse 1 . This corresponds to $\Delta \omega=10 \mathrm{THz}$, see text. The origin of the time corresponds to the moment when the center of the overlapped pulses reaches $z=0$. The laser intensity 
modulation moves downward in the direction of $\vec{K}_{g}$ with a phase velocity $v_{p}$. (b), Temporal evolution of the refractive index modulation at $z=0$ induced by the rotational Raman response. The refractive index modulation also moves downward. A temporal retardation with respect to the laser interference pattern is apparent at the beginning and end of the pulse overlap. (c), Temporal evolution of the plasma induced refractive index modulation at $z=0$. This plasma grating travels downward during its production process ( $-80 \mathrm{fs}$ to $40 \mathrm{fs}$ ). After $40 \mathrm{fs}$, the plasma density is constant because no decay mechanism is considered in the model. (d), Laser intensity (blue line, left ordinate), refractive index modulation due to Raman response (black line, right ordinate) and plasma (red line, right ordinate) at time $=-20 \mathrm{fs}$. The spatial phase shift between the laser intensity and the two index modulations is $1.2 \mu \mathrm{m}$ and $0.5 \mu \mathrm{m}$, respectively.
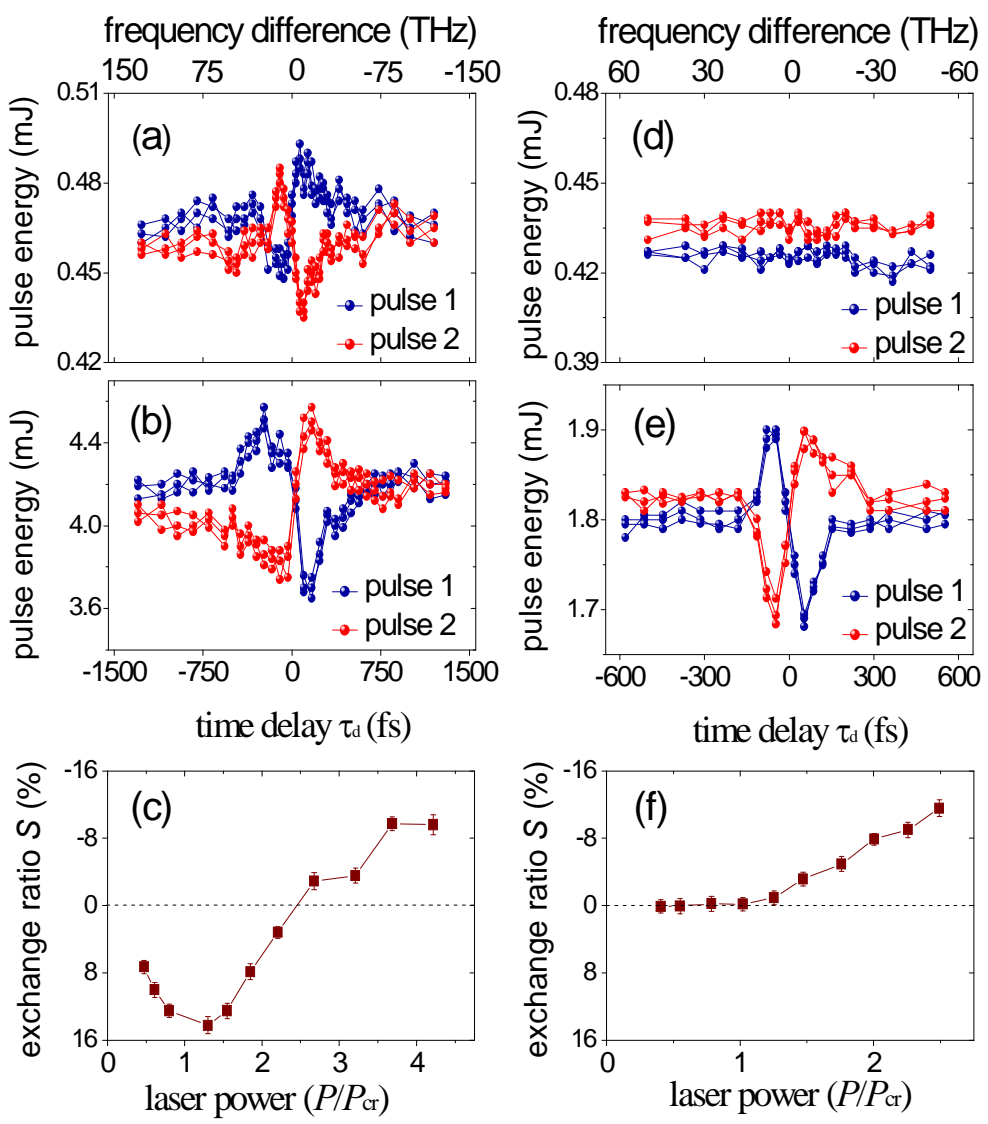

Fig. 3. Energy exchange of filaments in air (a, b, c) and argon (d, e, f). (a), Energy exchange of filaments in air as a function of delay (frequency difference $\Delta \omega$ ) between the chirped pulses with power $P=0.39 P_{\text {cr }}$ and duration $\tau_{\mathrm{p}}=220$ fs. Each point corresponds to the average from 100 measurements. (b), Same as in (a) except for $P=3.5 P_{\mathrm{cr}}$. (c), Maximum Energy exchange ratio $S$ recorded at positive delay $\tau_{\mathrm{d}}$ as a function of incident laser power in air. (d), (e), and (f), similar as (a), (b), (c), except air is replaced by Argon. In all cases, the focal lengths for both pulses are $2000 \mathrm{~mm}$ and the crossing angle is $0.9^{\circ}$. 

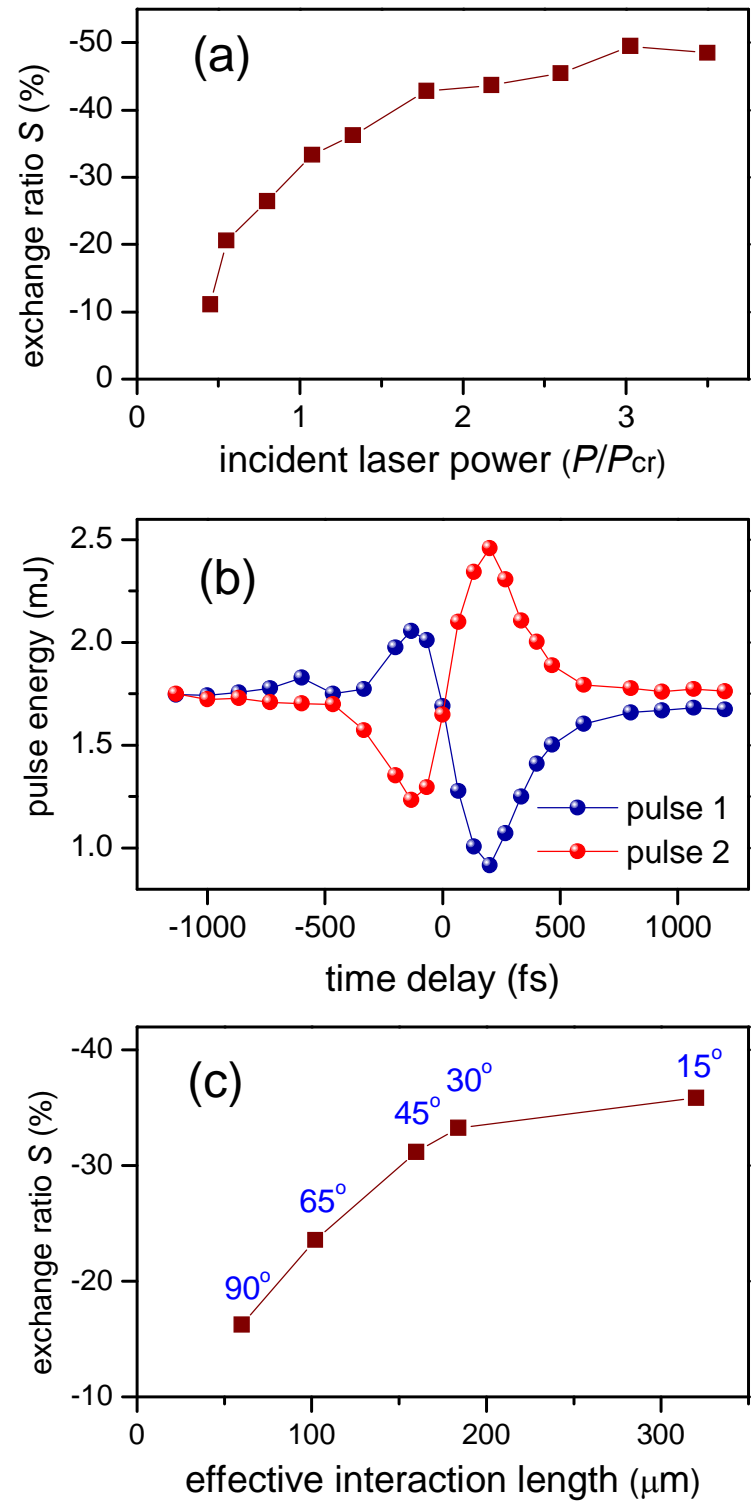

Fig. 4. (a) Ratio $S$ as a function of the incident laser power. The focal length for both pulses is $f=125 \mathrm{~mm}$. The crossing angle is $20^{\circ}$. Otherwise, same laser conditions as in Figure 3. (b) Typical energy exchange between two pulses with power of $1.5 P_{\mathrm{cr}}$. The experiment conditions are the same as in (a). (c), Ratio $S$ as a function of the interacting length $L$. The incident laser energy of both pulses is $1.15 P_{\mathrm{cr}}$. The focal length for both pulses is $f=$ $300 \mathrm{~mm}$ 